\title{
Interplay between the magnetic and transport properties in the III-V diluted magnetic semiconductor $\mathrm{Ga}_{1-x} \mathrm{Mn}_{x} \mathrm{As}$
}

\author{
A. Van Esch \\ Laboratorium voor Vaste-Stoffysica en Magnetisme, Katholieke Universiteit Leuven, B-3001 Leuven, Belgium \\ and IMEC, Kapeldreef 75, B-3001 Leuven, Belgium \\ L. Van Bockstal \\ Laboratorium voor Vaste-Stoffysica en Magnetisme, Katholieke Universiteit Leuven, B-3001 Leuven, Belgium \\ J. De Boeck \\ IMEC, Kapeldreef 75, B-3001 Leuven, Belgium \\ G. Verbanck \\ Laboratorium voor Vaste-Stoffysica en Magnetisme, Katholieke Universiteit Leuven, B-3001 Leuven, Belgium
}

A. S. van Steenbergen

Research Institute for Materials, High Field Magnet Laboratory, University of Nijmegen, 6525 ED Nijmegen, The Netherlands

P. J. Wellmann

Materials Department, University of California, Santa Barbara, California 93106

B. Grietens

IMEC, Kapeldreef 75, B-3001 Leuven, Belgium

R. Bogaerts and F. Herlach

Laboratorium voor Vaste-Stoffysica en Magnetisme, Katholieke Universiteit Leuven, B-3001 Leuven, Belgium

G. Borghs

IMEC, Kapeldreef 75, B-3001 Leuven, Belgium

(Received 17 March 1997; revised manuscript received 16 June 1997)

\begin{abstract}
Using a low-temperature molecular-beam epitaxy growth procedure, $\mathrm{Ga}_{1-x} \mathrm{Mn}_{x} \mathrm{As}$ - a III-V diluted magnetic semiconductor - is obtained with Mn concentrations up to $x \sim 9 \%$. At a critical temperature $T_{c}\left(T_{c}\right.$ $\approx 50 \mathrm{~K}$ for $x=0.03-0.05$ ), a paramagnetic to ferromagnetic phase transition occurs as the result of the interaction between $\mathrm{Mn}-h$ complexes. Hole transport in these compounds is strongly affected by the antiferromagnetic exchange interaction between holes and Mn $3 d$ spins. A model for the transport behavior both above and below $T_{c}$ is given. Above $T_{c}$, all materials exhibit transport behavior which is characteristic for systems near the metal-insulator transition. Below $T_{c}$, due to the rising spontaneous magnetization, spin-disorder scattering decreases and the relative position of the Fermi level towards the mobility edge changes. When the magnetization has reached its saturation value (below $\sim 10 \mathrm{~K}$ ) variable-range hopping is the main conduction mechanism. The negative magnetoresistance is the result of the expansion of the hole wave functions in an applied magnetic field. [S0163-1829(97)04044-7]
\end{abstract}

\section{INTRODUCTION}

Diluted magnetic semiconductors (DMS) are semiconductor alloys in which part of the lattice atoms has been substituted by a magnetic atom, thus inserting a local magnetic moment into the lattice. The most extensively studied and understood are based on II-VI materials in which a fraction of the group-II element has been replaced by manganese. ${ }^{1}$ Munekata et al. ${ }^{2}$ reported the first successful growth of a III-V DMS by molecular-beam epitaxy, namely $\mathrm{In}_{1-x} \mathrm{Mn}_{x}$ As. We have been able to grow near-perfect $\mathrm{Ga}_{1-x} \mathrm{Mn}_{x}$ As samples with high Mn concentrations by making use of a low-temperature growth procedure. III-V DMS containing Mn differ from their II-VI counterparts in that Mn not only introduces a magnetic moment into the lattice, it also functions as an acceptor. The coupling between the $\mathrm{Mn}$ magnetic core and the hole lies at the basis of the magnetic and magnetotransport behavior observed in these materials.

\section{SAMPLE PREPARATION AND STRUCTURAL ANALYSIS}

\section{A. Growth and post-growth annealing}

GaAs grown by molecular-beam epitaxy (MBE) at low temperature (LT) $\left(200-300{ }^{\circ} \mathrm{C}\right.$ instead of the normal growth temperature of $580-600{ }^{\circ} \mathrm{C}$ ) is excellent material with good 
crystal quality. When a growth procedure comparable to that of LT-GaAs is used, Mn concentrations up to $9 \%$ can be incorporated while retaining crystal homogeneity. ${ }^{3,4}$ All $\mathrm{Ga}_{1-x} \mathrm{Mn}_{x}$ As layers were grown on GaAs (100) substrates; a LT-GaAs buffer layer of typically $100 \mathrm{~nm}$ is deposited before a Mn flux is supplied to grow $\mathrm{Ga}_{1-x} \mathrm{Mn}_{x}$ As. The exact Mn concentration is determined after growth by energy dispersive x-ray analysis (EDS) or x-ray Bragg diffraction. Samples of $3 \mu \mathrm{m}$ thick with Mn concentrations up to $9 \%$ have been grown: the reflection high-energy electron diffraction pattern remained streaky throughout the whole growth procedure and the sample surfaces were smooth and shiny.

The as-grown samples were annealed for $30^{\prime \prime}$ at temperatures above the growth temperature to alter their magnetic and electrical properties. During the annealing, the sample surface is covered with a clean piece of GaAs to avoid As evaporation from the sample surface while a forming gas (a mixture of $10 \% \mathrm{H}_{2}$ and $90 \% \mathrm{~N}_{2}$ ) atmosphere suppresses oxidation during the annealing treatment.

A distinction should be made between two major annealing temperature regions. For temperatures slightly above the growth temperature (between 350 and $410{ }^{\circ} \mathrm{C}$ ) the amount of magnetically and electrically active $\mathrm{Mn}$ atoms in the $\mathrm{Ga}_{1-x} \mathrm{Mn}_{x}$ As lattice gradually reduces as the annealing temperature is increased. This was used to change $x$ continuously and in a controlled way in different pieces originating from one and the same growth, thus keeping all other parameters (buffer layer, interface quality, sample thickness, ... ) constant. When samples are submitted to temperatures above $500{ }^{\circ} \mathrm{C}$, ferromagnetic MnAs clusters start to form; initially small enough to exhibit superparamagnetic behavior but growing in size as the annealing temperature is increased up to $750^{\circ} \mathrm{C}$, above which the material starts to deteriorate and lose its magnetic properties. A study of these clustered systems has been published elsewhere., ${ }^{3,5}$ It should be stressed that by simply subjecting one sample to different annealing temperatures, a whole variety of physical systems can be studied, ranging from a homogeneous diluted magnetic semiconductor to nanoscale ferromagnetic precipitates embedded in a semiconductor matrix.

\section{B. Crystal structure}

MBE grown LT-GaAs epilayers have the zinc-blende crystal structure of GaAs $(a=5.6533 \AA)$, except that they contain 1-2\% excess arsenic, resulting in an expansion of the lattice parameter of the order of $0.094 \% .^{6,7}$ When submitted to annealing temperatures above $\sim 400{ }^{\circ} \mathrm{C}$, metallic As precipitates form and the lattice parameter evolves towards the substrate value of GaAs. Precipitates then reside in a near-perfect GaAs lattice.

As a rule, a ternary alloy formed by substituting Mn for the group-III element in the III-V lattice will retain the crystal structure of the parent III-V compound. ${ }^{1}$ The lattice parameters of all known DMS ternary alloys obey Vegard's law very closely; applied to $\mathrm{Ga}_{1-x} \mathrm{Mn}_{x}$ As it states

$$
a_{\mathrm{GaMnAs}}=(1-x) a_{\mathrm{LT}-\mathrm{GaAs}}+x a_{\mathrm{MnAs}},
$$

where $a_{\mathrm{LT}-\mathrm{GaAs}}=5.6572 \AA$ and $a_{\mathrm{MnAs}}=6.014 \AA^{2}$ is the lattice parameter of the hypothetical zinc-blende MnAs. The

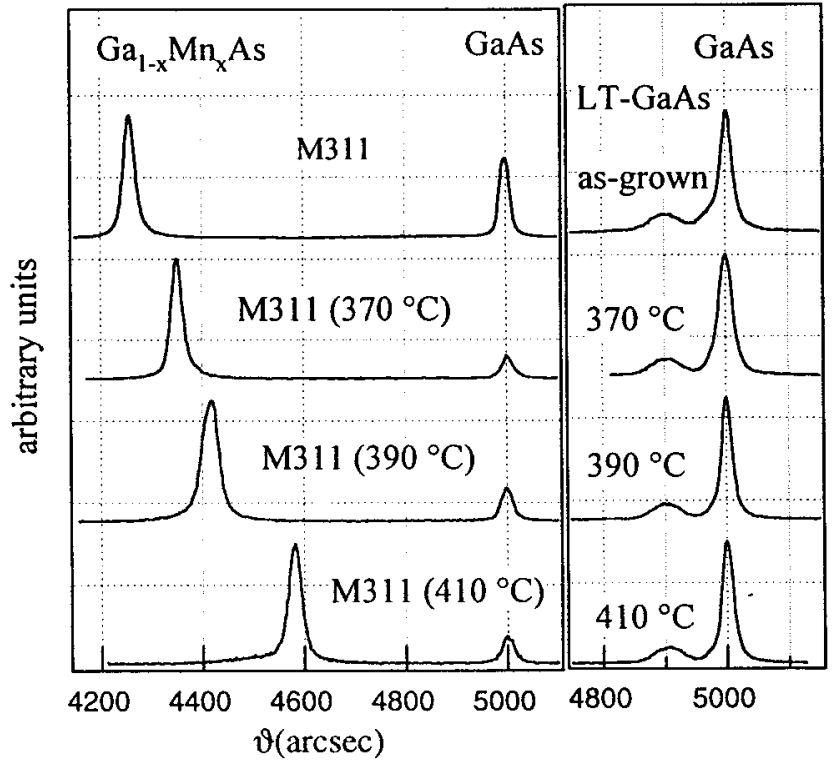

FIG. 1. X-ray-diffraction measurements on $\mathrm{Ga}_{0.913} \mathrm{Mn}_{0.087} \mathrm{As}$ (M311) and on a LT-GaAs reference sample, as-grown and after annealing. The annealing temperatures are indicated in the figure.

validity of Eq. (1) was confirmed by EDS measurements of $x$ in samples with a high Mn incorporation.

Subsequently, the lattice parameter-as determined by $\mathrm{x}$ ray diffraction-is used to calculate the Mn concentration $x$ for samples with lower concentrations $(x<2-3 \%)$ where EDS lacks accuracy and for samples submitted to lowtemperature annealing to determine the fraction of $\mathrm{Mn}$ remaining in the $\mathrm{Ga}_{1-} \mathrm{Mn}_{x}$ As lattice. In the latter $\mathrm{Mn}$ is extracted from the $\mathrm{Ga}_{1-x} \mathrm{Mn}_{x}$ As lattice (probably into Mn-As complexes) but not from the sample as a whole. The change in lattice parameter through the extraction of $\mathrm{Mn}$ from the DMS lattice is illustrated in Fig. 1, where $\theta-2 \theta$-scan data are shown for sample M311 submitted to different annealings. A LT-GaAs reference sample was grown and submitted to the same annealing treatments as the $\mathrm{Ga}_{1-x} \mathrm{Mn}_{x}$ As layers. As the $\theta-2 \theta$-scans (Fig. 1) show no changes in the reference layer for annealing temperatures below $410{ }^{\circ} \mathrm{C}$, we conclude that the presence of Mn enhances the mobility of the excess As atoms. From high resolution electron microscopy (HREM) pictures on multilayered structures of clustered $\mathrm{Ga}_{1-x} \mathrm{Mn}_{x} \mathrm{As} / \mathrm{GaAs} / \mathrm{AlAs}$ it can be inferred that As atoms are much more mobile than $\mathrm{Mn}$ atoms and diffuse preferentially towards the $\mathrm{Mn}$ atoms to form MnAs complexes. ${ }^{3}$ This conforms with the observations in Si $\delta$-doped LT-GaAs (Ref. 7) where it was found that doping lowers the onset temperature of cluster formation and excess As atoms preferentially migrate towards the impurity layer. An overview of the samples, their layer thickness, and Mn concentration is listed in Table I.

\section{Microscopic (local) structure of the DMS lattice}

While important from a crystallographic point of view, the local structure of $\mathrm{Mn}$ in the $\mathrm{Ga}_{1-x} \mathrm{Mn}_{x}$ As lattice also has a bearing on a number of other physical phenomena, e.g., on the sign of the charge carrier, the carrier concentration, and through this on the magnetic properties of the homogeneous DMS material. The microstructure of Mn doped GaAs layers 
TABLE I. Sample overview.

\begin{tabular}{lccc}
\hline \hline $\begin{array}{c}\text { Sample } \\
\text { name: }\end{array}$ & $\begin{array}{c}\text { Annealing } \\
\text { temperature }\end{array}$ & $x$ & $T_{c}$ \\
\hline $\mathrm{M} 271$ & & 0.060 & $62 \mathrm{~K}$ \\
$(0.4 \mu \mathrm{m})$ & $370{ }^{\circ} \mathrm{C}$ & 0.051 & $54 \mathrm{~K}$ \\
& $390{ }^{\circ} \mathrm{C}$ & 0.043 & $36 \mathrm{~K}$ \\
& $410{ }^{\circ} \mathrm{C}$ & 0.029 & $?$ \\
$\mathrm{M} 279$ & & 0.070 & $45 \mathrm{~K}$ \\
$(1.8 \mu \mathrm{m})$ & $370{ }^{\circ} \mathrm{C}$ & 0.061 & $29 \mathrm{~K}$ \\
& $390{ }^{\circ} \mathrm{C}$ & 0.038 & $16 \mathrm{~K}$ \\
$\mathrm{M} 311$ & & 0.087 & $38 \mathrm{~K}$ \\
$(2.3 \mu \mathrm{m})$ & $370{ }^{\circ} \mathrm{C}$ & 0.077 & $26 \mathrm{~K}$ \\
& $390{ }^{\circ} \mathrm{C}$ & 0.068 & $18 \mathrm{~K}$ \\
& $410{ }^{\circ} \mathrm{C}$ & 0.050 & $?$ \\
\hline \hline
\end{tabular}

has been investigated in the past for doping concentrations up to $10^{18}-10^{19} \mathrm{~cm}^{-3}$. Mn was found to reside on a Ga site in the zinc-blende structure $\left(\mathrm{Mn}_{\mathrm{Ga}}\right)$ taking up the acceptor function. The structure of the neutral $\mathrm{Mn}^{0}$ acceptor corresponds to the $3 d^{5}+$ hole configuration. ${ }^{7}$ As no ferromagnetic behavior is observed at room temperature, it can be assumed that the fraction of $\mathrm{Mn}$ atoms clustered into MnAs precipitates is negligible. All MBE grown (LT-) $\mathrm{Ga}_{1-x} \mathrm{Mn}_{x} \mathrm{As}$ samples show $p$-type conductivity, indicating that the majority of the Mn atoms find themselves in the acceptor function on the $\mathrm{Ga}$ lattice site $\left(\mathrm{Mn}_{\mathrm{Ga}}\right)$. However, from the discrepancy between x-ray diffraction measurements and magnetization or transport measurements on as-grown samples with increasing $\mathrm{Mn}$ incorporation, we conclude that selfcompensation is present. This discrepancy lies in the fact that an increased Mn concentration $x$ in the $\mathrm{Ga}_{1-x} \mathrm{Mn}_{x}$ As lattice does not necessarily imply an increased amount of magnetically and electrically active centers, as will be explained in the next paragraphs. No direct measurements on the crystallographic position of $\mathrm{Mn}$ in a $\mathrm{Ga}_{1-x} \mathrm{Mn}_{x}$ As lattice have been performed for $x>10^{19}-10^{20} \mathrm{~cm}^{-3}$, but compensation could come from Mn centers forming the small disordered sixfoldcoordinated centers with As $\left(\mathrm{Mn}^{6 \mathrm{As}}\right)$ as observed in the closely related $\operatorname{In}_{1-x} \mathrm{Mn}_{x} \mathrm{As}$ compound by Munekata et al. ${ }^{9,10}$ As these centers give rise to $n$-type conductivity, the degree of compensation is determined by the ratio $\mathrm{Mn}^{6 \mathrm{As}} / \mathrm{Mn}_{\mathrm{Ga}}$.

It may be useful to stress that this apparent decrease in Mn concentration through compensation differs from the extraction of the $\mathrm{Mn}$ atoms from the actual $\mathrm{Ga}_{1-x} \mathrm{Mn}_{x}$ As lattice through low-temperature annealing. In the former, part of the $\mathrm{Mn}$ atoms in an acceptor position are compensated by their donor counterparts. All Mn atoms have however taken up a site in the $\mathrm{Ga}_{1-x} \mathrm{Mn}_{x}$ As lattice. When submitted to temperatures above the growth temperature, the excess As atoms in the lattice become more mobile and exclude Mn centers from the $\mathrm{Ga}_{1-x} \mathrm{Mn}_{x}$ As lattice by incorporating them into Mn-As complexes and eventually into MnAs ferromagnetic clusters.

These results are not in contradiction with the x-ray diffraction data which predict Vegard's law behavior for the ternary lattice. X-ray diffraction is not sensitive to the topological details surrounding an individual lattice point, but

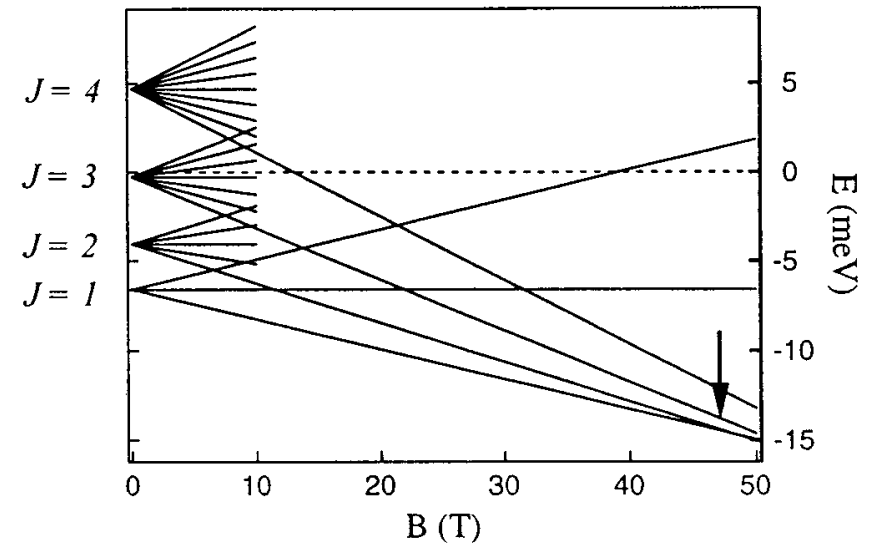

FIG. 2. Splitting of the energy levels of an isolated Mn- $h$ complex with total spin $J$ in a magnetic field. The dashed line is the zero level, i.e., when no magnetic interaction is present. The arrow marks the crossing of the $J=1$ and 2 level at $\sim 48 \mathrm{~T}$.

determines the lattice parameter $a$ averaged over many anion and cation sites. It is the averaged $a(x)$ which varies linearly with $x$.

\section{Magnetic state of the hole bound to the manganese ion}

In the most accepted model for a Mn impurity on a $\mathrm{Ga}$ site in the GaAs lattice $\mathrm{Mn}$, being one electron short to fit into the III-V bonding, takes up an acceptor function. ${ }^{8,11-13}$ The binding energy of the hole bound to the acceptor is 113 $\mathrm{meV}$ and the Bohr radius is $\sim 10 \AA$. The electronic structure of the neutral Mn acceptor in GaAs, derived from electronspin-resonance (ESR) measurements, was described by Schneider et $a l .{ }^{8}$ The ground state is formed by the antiferromagnetic coupling between the $S=\frac{5}{2}$ spin of the manganese core and the $j=\frac{3}{2}$ spin of the hole to form a manganesehole $(\mathrm{Mn}-h)$ complex with total angular momentum $J=1$ $(\mathbf{J}=\mathbf{S}+\mathbf{j})$. Excited states with $J=2,3$, and 4 are formed at energies $2 \epsilon, 5 \epsilon$, and $9 \epsilon, \epsilon$ being the exchange coupling. The experimental values $2 \epsilon=2.5 \pm 0.5 \mathrm{meV}$ and $g_{J=1}=2.77$ are determined from ESR and susceptibility in combination with magnetization measurements. ${ }^{14,15}$ In a magnetic field, each $J$ level splits up into $2 J+1$ levels. Figure 2 shows the dependence of the energy levels on the magnetic field. The reference level is the one where no magnetic interaction is present. Crossing of the $J=1$ with the $J=2$ level takes place in a field of nearly $50 \mathrm{~T}$. The $J=1$ state can be considered the sole state contributing to the low-temperature behavior (below $\sim 10 \mathrm{~K}$ ) of $\mathrm{Ga}_{1-x} \mathrm{Mn}_{x}$ As for all fields considered in this paper.

\section{MAGNETIC PROPERTIES}

Below a transition temperature $T_{c}$ dependent on the $\mathrm{Mn}$ concentration, the $\mathrm{Ga}_{1-x} \mathrm{Mn}_{x}$ As layers become ferromagnetic. Magnetization measurements in a very low magnetic field $(0.2 \mathrm{mT})$ were performed in a SQUID (Fig. 3) and clearly show the onset of magnetic ordering at $T_{c}=45 \mathrm{~K}$ for M279. In contrast to the commonly observed magnetic spin-glass phase transition in II-VI DMS (Ref. 1) the 


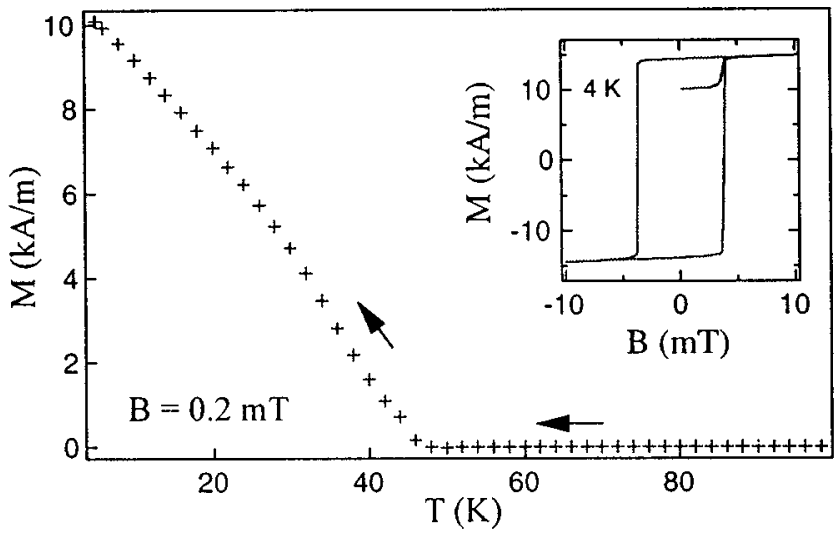

FIG. 3. Magnetization measurements performed on $\mathrm{Ga}_{0.930} \mathrm{Mn}_{0.070} \mathrm{As}$ (M279) in a SQUID in a magnetic field of $0.2 \mathrm{mT}$ show the onset of magnetic ordering at $T_{c}=45 \mathrm{~K}$. The inset shows the hysteresis measured at $T=4 \mathrm{~K}$ with the field parallel to the surface.

phase transition in $\mathrm{Ga}_{1-x} \mathrm{Mn}_{x} \mathrm{As}$ is ferromagnetic: acsusceptibility measurements (Fig. 4) show no difference between zero-field-cooled and field-cooled data and no frequency dependence.

At $4 \mathrm{~K}$ a hysteresis loop with a saturation magnetization of $15 \mathrm{kA} / \mathrm{m}$ is observed for $\mathrm{Ga}_{0.93} \mathrm{Mn}_{0.070} \mathrm{As}$ (M279). The coercive field parallel to the sample surface is $3200 \mathrm{kA} / \mathrm{m}$. The saturated magnetic moment at $4 \mathrm{~K}$ shows a discrepancy with the concentrations determined by $\mathrm{x}$-ray analysis. From the saturation magnetization $M=\mu_{B} g_{J=1} J N_{\mathrm{Mn}} / V \quad[$ $g_{J=1}=2.77$ and $J=1$ (Refs. 8 and 16)] about $40 \%$ of the Mn in the $\mathrm{Ga}_{0.93} \mathrm{Mn}_{0.070} \mathrm{As}$ (M279) lattice is estimated to contribute to the ferromagnetic behavior. As mentioned above this is due to the fact that a fraction of the $\mathrm{Mn}$ atoms forms the small disordered sixfold-coordinated centers with $\mathrm{As}\left(\mathrm{Mn}^{6 \mathrm{As}}\right)$ as observed in $\mathrm{In}_{1-x} \mathrm{Mn}_{x}$ As. As these centers take up a donor

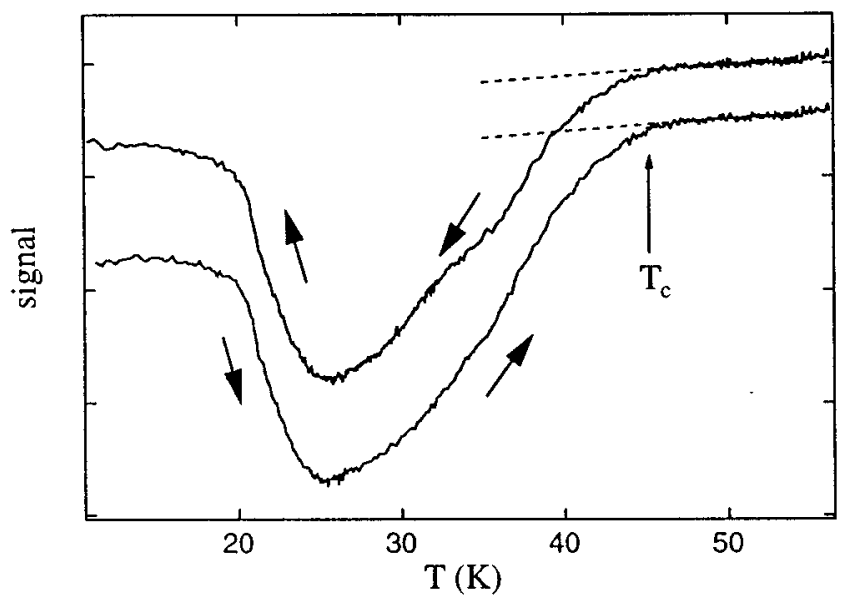

FIG. 4. ac-susceptibility measurements on $\mathrm{Ga}_{0.930} \mathrm{Mn}_{0.070} \mathrm{As}$ (M279) as a function of temperature show no difference between field-cooled and zero-field-cooled data $(B=10 \mathrm{mT})$. The fieldcooled data are displaced for clarity. $T_{c}=45 \mathrm{~K}$ is marked in the figure.

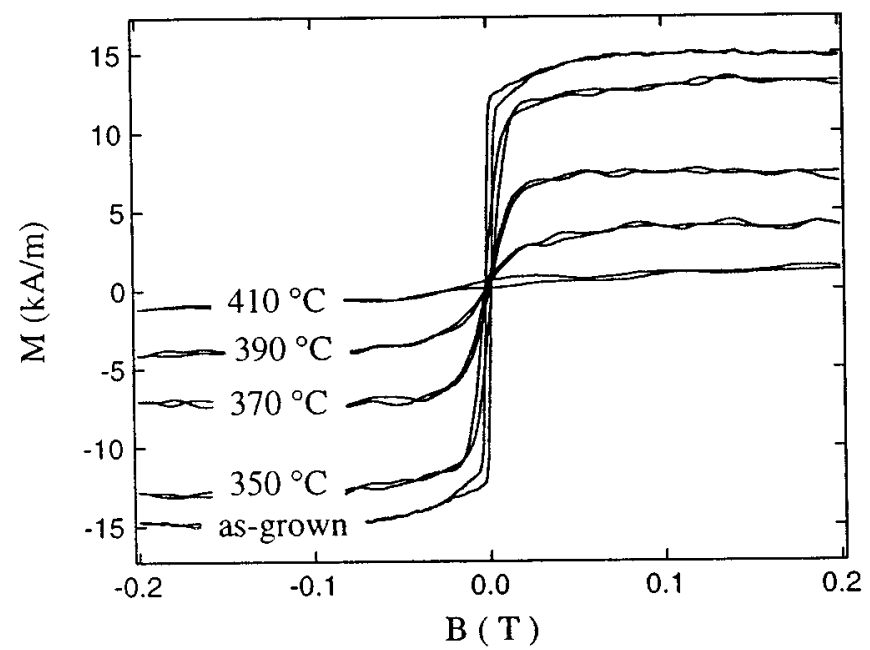

FIG. 5. Magnetization measurements at $T=10 \mathrm{~K}$ on $\mathrm{Ga}_{0.913} \mathrm{Mn}_{0.087} \mathrm{As}$ (M311) show a decrease in saturation magnetization after low-temperature annealing. The annealing temperatures are indicated in the figure.

function, it can be estimated that in M279 about 30\% of the incorporated $\mathrm{Mn}$ atoms reside on this $\mathrm{Mn}^{6 \mathrm{As}}$ site, compensating $\mathrm{Mn}$ atoms on a substitutional lattice site. Only the uncompensated $\mathrm{Mn}$ atoms can form a Mn- $h$ complex and thus contribute to the ferromagnetic behavior. $T_{c}$ increases with Mn concentration up to $x \sim 5 \%$; excess incorporation of $\mathrm{Mn}$ only adds to the amount of $\mathrm{Mn}^{6 \mathrm{As}}$ centers, resulting in a lower net amount of $\mathrm{Mn}-h$ complexes and consequently a decrease in $T_{c}$. This hypothesis is further supported by the transport behavior where a lowering of the Fermi level relative to the mobility edge is observed for increasing $\mathrm{Mn}$ incorporation (above $\sim 5 \%$ ), as discussed in the next paragraph. It is already obvious that the hole in the $\mathrm{Mn}-h$ complex plays the crucial role in mediating the ferromagnetic interaction.

Post-growth annealing reduces the amount of magnetically (and electrically) active Mn atoms as illustrated in Fig. 5, where M311 has been submitted to different annealing temperatures. The extraction of Mn through annealing differs from the mechanism of compensation as explained in the previous paragraph. For the highest Mn incorporations it is not unlikely that a fraction of the $\mathrm{Mn}$ atoms in the as-grown material is absorbed in Mn-As complexes, but this is accounted for by the use of $\mathrm{x}$-ray diffraction to calculate the actual value of $x$ in $\mathrm{Ga}_{1-x} \mathrm{Mn}_{x}$ As.

Magnetization measurements at $4 \mathrm{~K}$ with a torque magnetometer in a magnetic field up to $20 \mathrm{~T}$ (Fig. 6) reveal that the saturation magnetization is fully reached at a very low field and does not increase anymore beyond that. The measurements were performed on three different samples, each with a different $T_{c}$. The diamagnetic background from the GaAs substrate was measured on a reference GaAs sample and then substracted from the $\mathrm{Ga}_{1-x} \mathrm{Mn}_{x}$ As signal. The results shown in Fig. 6 are unambiguous: the linear behavior of the torque as a function of the applied field illustrates the constant magnetization. This is an important fact because it makes the model of the partially saturated bound magnetic polaron as suggested by Ohno et al. ${ }^{16}$ for $\operatorname{In}_{1-x} \mathrm{Mn}_{x}$ As inap- 


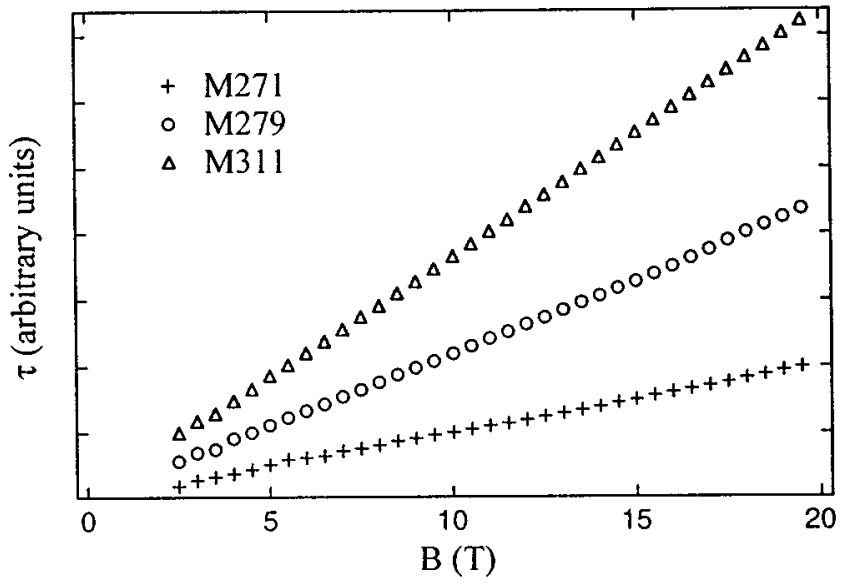

FIG. 6. Torque measurements in fields up to $20 \mathrm{~T}$ on $\mathrm{Ga}_{0.940} \mathrm{Mn}_{0.060} \mathrm{As} \quad(\mathrm{M} 271), \quad \mathrm{Ga}_{0.930} \mathrm{Mn}_{0.070} \mathrm{As} \quad$ (M279), and $\mathrm{Ga}_{0.913} \mathrm{Mn}_{0.087} \mathrm{As}$ (M311). The linear behavior corresponds to a constant magnetization.

plicable to $\mathrm{Ga}_{1-x} \mathrm{Mn}_{x} \mathrm{As}$. Ohno et al. explain the negative magnetoresistance observed in $\operatorname{In}_{1-x} \mathrm{Mn}_{x}$ As through the formalism of unsaturated bound magnetic polarons having partially aligned spins in zero field. Alignment of the canted spins in a magnetic field could then be responsible for the negative magnetoresistance. The high-field magnetization data exclude that this is also the case for $\mathrm{Ga}_{1-x} \mathrm{Mn}_{x} \mathrm{As}$; the negative magnetoresistance at low temperatures cannot be explained by an increased magnetization. The high-field magnetization data show no contribution from the Mn atoms not participating in the ferromagnetic phase.

The observed temperature dependence of the spontaneous magnetization in $\mathrm{Ga}_{1-x} \mathrm{Mn}_{x} \mathrm{As}$ (Fig. 7) differs from the universal behavior of ferromagnets ${ }^{17}$ (represented by the dashed

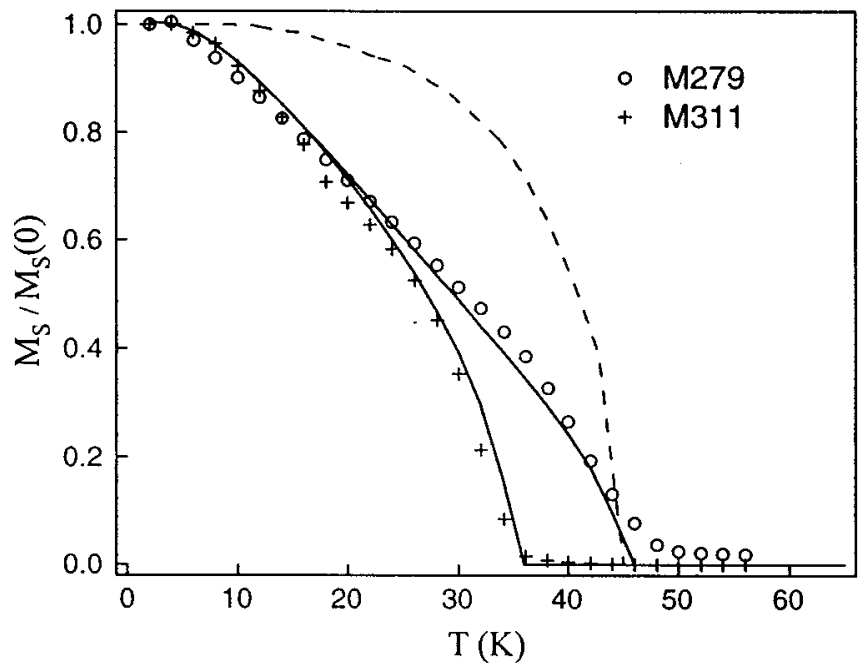

FIG. 7. The saturation magnetization $\left[\mathrm{Ga}_{0.930} \mathrm{Mn}_{0.070} \mathrm{As}\right.$ (M279) and $\mathrm{Ga}_{0.913} \mathrm{Mn}_{0.087} \mathrm{As}$ (M311)] as a function of temperature. The dashed line represents the universal behavior of ferromagnets resulting from a single ferromagnetic exchange interaction. The solid lines are fits based on the mean-field theory extended to multiple exchange mechanisms. line in Fig. 7) because it is the result of multiple exchange interactions. A qualitative understanding of this behavior can be gained by extending the mean-field theory, incorporating different exchange mechanisms. First, there is the coupling between the spin $S$ of a Mn core and the spin $j$ of the hole surrounding it, known to be antiferromagnetic. Second, assuming the holes to be responsible for the ferromagnetic ordering, the interaction between the hole spins results in a parallel alignment and is called ferromagnetic. The direct exchange interaction between two Mn cores is considered negligible. The Hamiltonian describing the magnetic energy of one hole with spin $j$ is

$$
H^{h}=-\sum_{l} J_{l}^{\mathrm{hh}} \mathbf{j} \cdot \mathbf{j}_{l}-J^{\mathrm{Mnh}} \mathbf{j} \cdot \mathbf{S}+g_{h} \mu_{B} \mathbf{j} \cdot \mathbf{B},
$$

where $\mathbf{j}, \mathbf{j}_{l}$, and $\mathbf{S}$ are the spin operators for the holes and the $\mathrm{Mn}$ core, respectively, $J^{\mathrm{hh}}$ is the hole-hole exchangecoupling constant $\left(J^{\mathrm{hh}}>0\right)$ and $J^{\mathrm{Mnh}}$ is the Mn-hole exchange-coupling constant $\left(J^{\mathrm{Mnh}}<0\right)$. In the second term we have assumed that the Mn spin $S$ only interacts with the one hole surrounding it. When we restrict the summation in the first term to the nearest neighbors instead of all lattice sites occupied by a Mn- $h$ complex and assume $J^{\text {hh }}$ to be constant for all nearest-neighbor pairs, in the mean-field approximation the magnetic energy of the hole becomes

$$
E^{h}=\left[-q J^{\mathrm{hh}}\left\langle j_{z}\right\rangle-J^{\mathrm{Mnh}}\left\langle S_{z}\right\rangle+g_{h} \mu_{B} B\right] j_{z},
$$

$q$ is the number of nearest-neighbor holes. In analogy to the mean-field theory the degree of polarization of the hole spin is

$$
\left\langle j_{z}\right\rangle=\frac{3}{2} \mathcal{B}_{j}\left(\frac{\left(-q J^{\mathrm{hh}}\left\langle j_{z}\right\rangle-J^{\mathrm{Mnh}}\left\langle S_{z}\right\rangle+g_{h} \mu_{B} B\right) j}{k T}\right),
$$

where $\mathcal{B}_{j}$ is the Brillouin function. The Hamiltonian describing the magnetic energy of a Mn core can be written

$$
H^{\mathrm{Mn}}=-J^{\mathrm{Mnh}} \mathbf{j} \cdot \mathbf{S}+g_{\mathrm{Mn}} \mu_{B} \mathbf{S} \cdot \mathbf{B},
$$

with $g_{\mathrm{Mn}}=2$. Making the approximations as stated above, the expression for $\left\langle S_{z}\right\rangle$ becomes

$$
\left\langle S_{z}\right\rangle=\frac{5}{2} \mathcal{B}_{S}\left(\frac{\left(-J^{\mathrm{Mnh}}\left\langle j_{z}\right\rangle+g_{\mathrm{Mn}} \mu_{B} B\right) S}{k T}\right) .
$$

Equations (4) and (6) form a set of equations that can be solved numerically to obtain the magnetization as a function of temperature. Applied to the experimental data presented in Fig. 7, the obtained fitvalues for the exchange constants are $q J^{\mathrm{hh}}=1.0 \mathrm{meV}$ for $\mathrm{M} 311, q J^{\mathrm{hh}}=2.2 \mathrm{meV}$ for $\mathrm{M} 279$, and $J^{\mathrm{Mnh}}=-1.2 \mathrm{meV}$ for both samples, in excellent agreement with the value of the exchange constant as determined for dilute systems $\left(J^{\mathrm{Mnh}} \approx-\epsilon\right)$. The strength of the ferromagnetic interaction, given by $q J^{\mathrm{hh}}$, is smallest for M311, in agreement with the lower amount of magnetically active $\mathrm{Mn}-h$ complexes. 


\section{TRANSPORT PROPERTIES}

\section{A. The impurity band}

For high doping concentrations, impurity states belonging to different centers merge into an impurity band of finite width in energy. Depending on the doping (and compensation), the electronic states of the impurity band may be localized or delocalized. ${ }^{18-20}$ If the Fermi level is well in the localized-state region, conduction at low temperatures is due to carrier hopping and is exponentially dependent on temperature and impurity concentration. The material is considered an insulator. If the Fermi level is in the extended region, conduction remains finite at zero temperature and the material is called metallic. The transition between both regimes is called the metal-insulator transition (MIT) and the energy level separating the localized from the extended states has been named the "mobility edge"' $E_{m}$.

The band structure and relative positions of the Fermi level and mobility edge are estimated in the following calculations. The bandwidth $W$ is given by

$$
W=2 q I,
$$

where $q$ is the coordination number and the overlap integral $I$ between two sites at a distance $R$ apart can be written as ${ }^{18}$

$$
I=\left[\frac{3}{2}(1+\alpha R)+\frac{1}{2}(\alpha R)^{2}\right] \frac{e^{2} \alpha}{\kappa} \exp (-\alpha R),
$$

where $\alpha=1 / a_{0}$ ( $a_{0}$ being the effective Bohr radius of the isolated wave function) and $\kappa$ is the dielectric constant of GaAs. The middle of the band lies at an energy

$$
\frac{1}{2} \frac{e^{2} \alpha}{\kappa}
$$

above the valence band ( $\approx 110 \mathrm{meV}$ for $\mathrm{Mn}$ in $\mathrm{GaAs})$. It can easily be calculated that merging occurs for $\alpha R \approx 2.55$, corresponding to $x \approx 0.002$.

When $4.9 \%$ of the $\mathrm{Ga}$ atoms have been replaced by a $\mathrm{Mn}$ atom (as is the case for M279), a half bandwidth of $215 \mathrm{meV}$ is obtained. The impurity band, however, is only partially filled (represented by the shaded region) because of the compensation. From the saturation magnetization measurements, it was estimated that, for M279, only $60 \%$ of the band is filled (at $T=0 \mathrm{~K}$ ). An energy diagram is sketched in Fig. 8.

It may be useful to mention that, although the MIT is generally related to the overlap of the lower and upper Hubbard band, ${ }^{18,20}$ we have only considered merging of the impurity band (or lower Hubbard band) with the valence band. We have done so because the coupling of the carriers with the magnetic Mn centers enhances the separation between the two Hubbard bands: the energy required to take a hole from one of the centers and put it on a distant already occupied one is now considerably larger than just the Coulomb energy separating the two Hubbard bands in the MottHubbard-Anderson model. ${ }^{20}$ The MIT in our model is completely analogous to the MIT as described in the MottHubbard-Anderson model, except that the valence band takes the role of the upper Hubbard band. An important consequence is that both the critical concentration at which the MIT takes place and the minimum metallic conductivity are
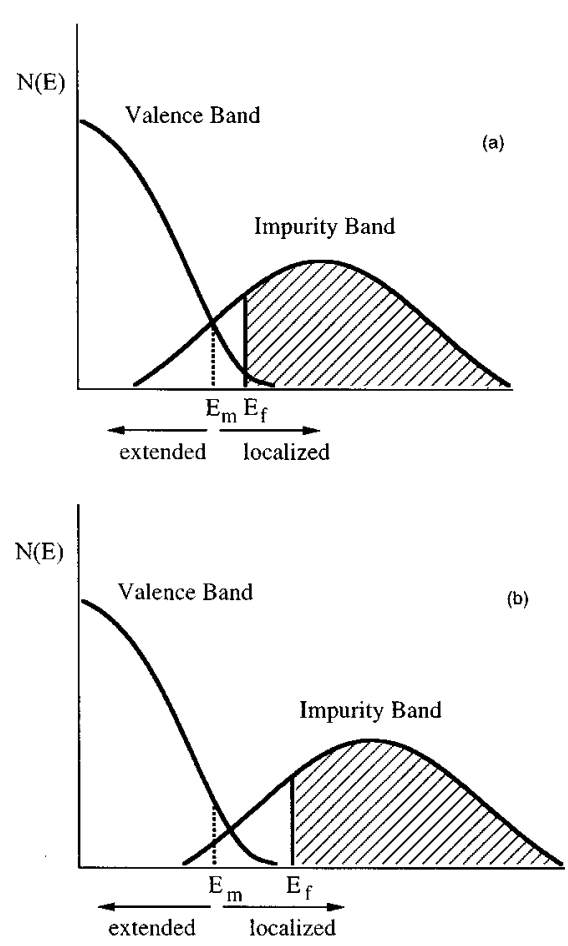

FIG. 8. Schematic representation of the band structure in the $\mathrm{Ga}_{1-x} \mathrm{Mn}_{x} \mathrm{As}$ compound with $x \gg 0.2 \%$. The shaded regions indicate the occupied states at $T=0 \mathrm{~K}$. (a) Above $T_{c}$, conduction occurs through thermal excitation of holes to states below $E_{m}$. (b) Below $T_{c}$ the localization has increased and the main contribution to the conductivity comes from hopping conduction between localized states.

larger than would be expected from the Mott criterion (i.e., $R$ decreases by a factor of two $\left.{ }^{18}\right)$ :

$$
\sigma_{\min }=C \frac{e^{2}}{\hbar R_{\text {crit }}},
$$

with $C \approx 0.1$ and $R_{\text {crit }}=25.5 \AA$ as calculated above, $\sigma_{\mathrm{mm}}$ is about $100(\Omega \mathrm{cm})^{-1}$.

\section{B. High-temperature behavior: $T>T_{c}$}

Above $T_{c}$, resistivity measurements (Fig. 9) show a transport behavior characteristic for systems near the metalinsulator transition, where the resistivity is inversely proportional with temperature. Dubson and Holcomb ${ }^{21}$ derived the temperature dependence of the conductivity near the MIT point from the energy dependence of the zero-temperature conductivity properties around the Fermi level. When applied to hole transport, this temperature dependence is given by

$$
\sigma(T) \sim k T \ln \left[1+\exp \left(\frac{E_{m}-E_{f}}{k T}\right)\right] .
$$

This equation is valid only within a critical range around the MIT: deep on the insulating side of the transition we expect hopping conduction to dominate and on the metal side of the transition, the assumptions behind Eq. (11) break down for $\sigma>\sigma_{\min }$. Above $T_{c}$, all samples studied in this paper fall within this critical region. 


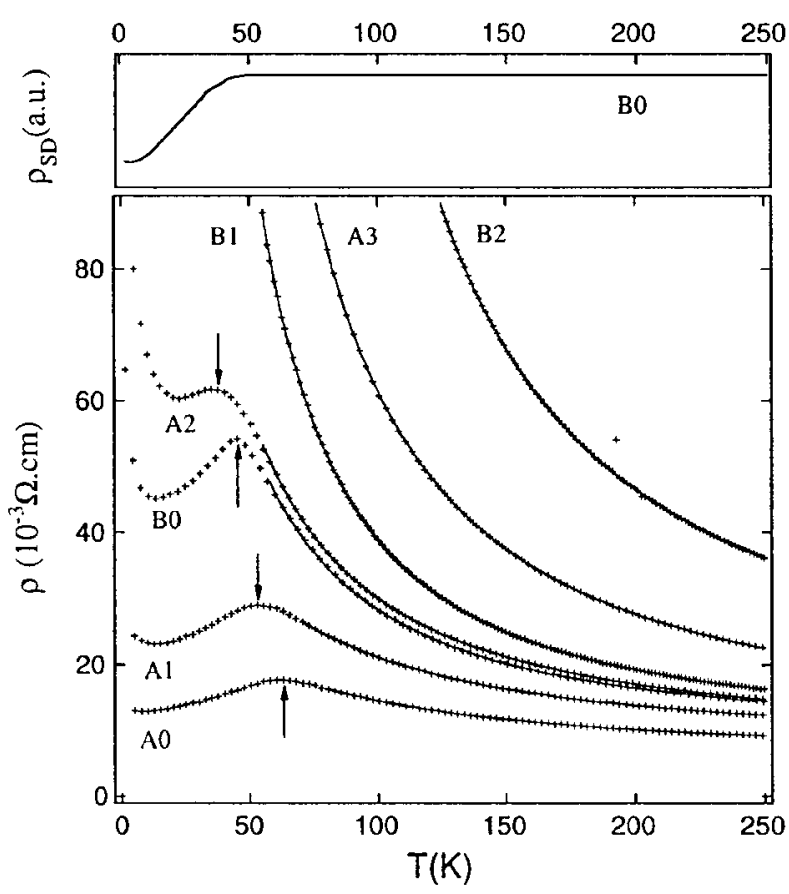

FIG. 9. Resistivity measurements on $\mathrm{Ga}_{0.940} \mathrm{Mn}_{0.060} \mathrm{As}$ (M271) $(A)$ and $\mathrm{Ga}_{0.930} \mathrm{Mn}_{0.070} \mathrm{As}$ (M279) (B), as-grown ( $A 0$ and $\left.B 0\right)$, and after annealing $\left(A 1\right.$ and $B 1$ were annealed at $370{ }^{\circ} \mathrm{C}, A 2$ and $B 2$ were annealed at $390{ }^{\circ} \mathrm{C}, A 3$ was annealed at $\left.410^{\circ} \mathrm{C}\right)$. Fits are shown for the high-temperature $\left(T>T_{c}\right)$ behavior. The arrows indicate the paramagnetic to ferromagnetic phase transition. The top part of the figure shows the effect of the spin-disorder scattering on the resistivity $\left(\rho_{\mathrm{SD}}\right)$, as calculated for M279 (B0) from magnetization data.

According to Eq. (11), the low-temperature conductivity close to the metal-insulator transition becomes

$$
\begin{gathered}
\sigma(T \rightarrow 0) \sim k T \exp \frac{E_{m}-E_{f}}{k T} \text { for }\left(E_{m}-E_{f}\right)<0, \\
\sigma_{\mathrm{MIT}} \sim k T \ln 2 \text { for }\left(E_{m}-E_{f}\right)=0, \\
\sigma(T \rightarrow 0) \sim\left(E_{m}-E_{f}\right) \quad \text { for }\left(E_{m}-E_{f}\right)>0 .
\end{gathered}
$$

On the insulator side $\left[\left(E_{m}-E_{f}\right)<0\right.$ for hole transport], transport at low temperatures approaches the activated behavior typical for insulating materials. The temperature dependence of the conductivity at the MIT is linear. On the metal side $\sigma(T \rightarrow 0)$ tends towards the low-temperature conductivity given by Abrahams et al. ${ }^{22}$ when the critical exponent is approximately 1 .

Equation (11) is calculated based on the zero-temperature conductivities for different positions of the Fermi level and neglects all temperature-dependent effects other than those arising from the occupation of the energy levels (i.e., the conductivity is strictly proportional to the amount of charge carriers above the mobility edge when a constant mobility and a constant density of states are assumed). Including the temperature dependence of phonon scattering and reverting it to resistivity, Eq. (11) becomes

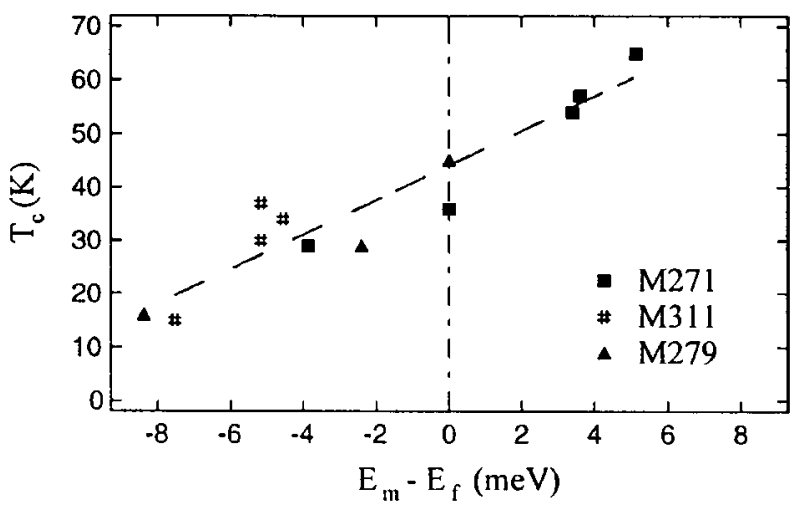

FIG. 10. Curie temperature versus $E_{m}-E_{f}$ (above $T_{c}$ ), as obtained from fits on the resistivity data.

$$
\rho(T)=\frac{C_{1}+F}{k T \ln \left(1+\exp \frac{E_{m}-E_{f}}{k T}\right)},
$$

with

$$
F=\frac{C_{2}}{\exp \frac{\theta_{D}}{T}-1},
$$

where $F$ represents phonon scattering $\left(C_{2}\right.$ is a constant), $C_{1}$ represents the temperature-independent part of the mobility, and $\theta_{D}$ is the Debye temperature. All fitted resistivity curves showed a Debye temperature close to $385 \mathrm{~K}$ in agreement with the phonon frequency in GaAs. The sign of $\left(E_{m}-E_{f}\right)$ obtained from the fit reveals whether the material is on the insulating or metallic side of the MIT. From Fig. 10 it is evident that by lowering the active Mn content (either by changing the effusion flux during growth or by post-growth annealing), $\mathrm{Ga}_{1-x} \mathrm{Mn}_{x}$ As can be tuned through the metalinsulator transition. M311 (for all annealing treatments), M271 annealed at $410{ }^{\circ} \mathrm{C}$, and M279 annealed at $370{ }^{\circ} \mathrm{C}$ are on the insulator side, M279 (as-grown) and M271 annealed at $390{ }^{\circ} \mathrm{C}$ are exactly at the MIT point and all other samples are metallic. The annealing causes an increase in overall resistivity; it reduces the amount of $\mathrm{Mn}-h$ complexes (cf. Fig. 5) changing both $\left(E_{m}-E_{f}\right)$ and the Curie temperature. This decrease in $T_{c}$ as a function of annealing temperature is again proof of the crucial role played by the average distance between the magnetically active centers (i.e., by the overlap of the hole orbitals) in the ferromagnetic behavior of the system.

\section{Low-temperature behavior: $T<T_{c}$}

Below $T_{c}$ the paramagnetic to ferromagnetic transition is initially accompanied by a decrease in resistivity (Fig. 9). For sufficiently low temperatures, the resistivity increases. This behavior is the result of changes in both the spindisorder scattering and the conduction mechanism. The local maximum occurs exactly at the Curie temperature, as can be seen from comparing magnetization (Figs. 3, 4, or 7) and resistivity data on M279 (curve $B 0$ in Fig. 9). The initial drop in resistivity below $T_{c}$ is due to an increase in the carrier mobility, as is observed in ferromagnetic 
semiconductors. ${ }^{23}$ The result of a perturbation calculation of the spin-disorder scattering on the resistivity in metals $\left(\rho_{\mathrm{SD}}\right)$, but neglecting short-range order, is given by ${ }^{23}$

$$
\begin{aligned}
\rho_{\mathrm{SD}}= & \frac{m^{* 2} k_{f} N}{\pi n e^{2} \hbar^{3}} J^{2}\left[S(S+1)-S^{2}\left(\frac{M}{M_{0}}\right)^{2}\right. \\
& \left.-S\left(\frac{M}{M_{0}}\right) \tanh \left(\frac{3 T_{c} M}{2 T S(S+1) M_{0}}\right)\right] .
\end{aligned}
$$

Applied to the observed magnetization in the $\mathrm{Ga}_{1-x} \mathrm{Mn}_{x} \mathrm{As}$ system, $\rho_{\mathrm{SD}}$ varies as shown in the top part of Fig. 9. The spin-disorder scattering remains constant in the paramagnetic region, below $T_{c}$ it drops gradually until the magnetic order of the spin system is complete. Short-range order will cause a sharp minimum in the mobility at $T_{c}$, due to critical scattering. ${ }^{23}$ Fits on the resistivity data, however, show that no such sharp minimum is present and the magnetic contribution in the carrier mobility can be regarded as a constant above $T_{c}$. Below $\sim 10 \mathrm{~K}$, the magnetization has reached its saturation value and the spin-disorder scattering does not change anymore with decreasing temperature.

As in traditional ferromagnetic semiconductors, the increasing magnetization causes a splitting of both the valence band and the impurity band. In conventional ferromagnetic semiconductors the splitting of the impurity band is always smaller than that of the valence band as the acceptor (or donor) level never originates from the magnetic element; below $T_{c}$ the activation energy decreases. This no longer holds for $\mathrm{Ga}_{1-x} \mathrm{Mn}_{x} \mathrm{As}$. On the contrary, the acceptor level is now strongly correlated to the magnetic center. Hence, the energy to excite a hole from the impurity band to a nonlocalized state (in the valence band) increases as the magnetization increases. This is sketched in Fig. 8(b). Below $\sim 10 \mathrm{~K}$ (depending on the Mn concentration) the magnetization has reached its saturation magnetization and $\rho_{\mathrm{SD}}$ remains constant. Resistivity measurements reveal variable-range hopping, characterized by Mott's law: ${ }^{18,19}$

$$
\rho(T)=\rho_{0} \exp \left[\left(\frac{T_{0}}{T}\right)^{1 / 4}\right]
$$

The observation that the main contribution to the electrical conductivity comes from holes hopping directly between impurities (without excursion to the valence band) is illustrated in Fig. 11. As this also applies to samples that were found to be on the metallic side of the MIT above $T_{c}$, the presumption that localization increases when ferromagnetism sets in is confirmed [Fig. 8(b)]. The hopping mechanism corresponds to a very low mobility, since it is associated with the overlap of wave-function tails. Nevertheless, when the Fermi level is in the localized region hopping wins in the competition with band conduction at low temperatures as the activation energies of the former are very low. The compensation inherently present in all samples provides the necessary empty acceptor sites to allow hopping within the impurity band. The effect of post-growth annealing on the slope of $\ln \rho$ vs $T^{1 / 4}$ is in agreement with the fact that the activation energy increases when the mean distance between hopping centers increases.

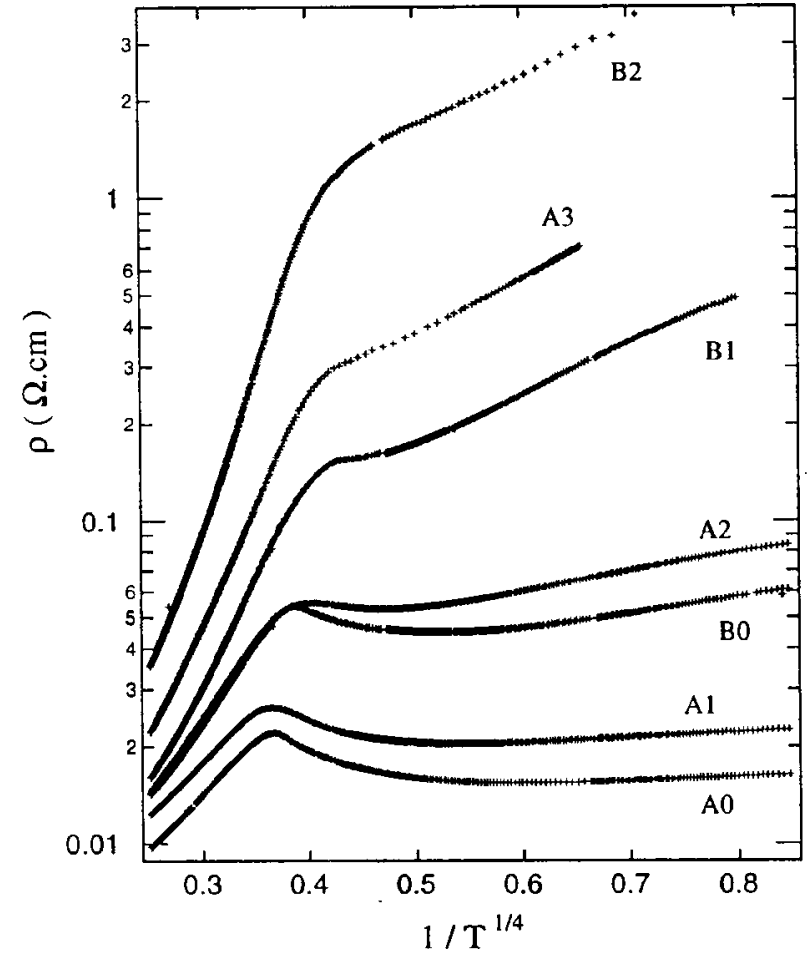

FIG. 11. Resistivity data (as displayed in Fig. 8) plotted as a function of $T^{-1 / 4}$ reveal variable-range hopping as the lowtemperature transport mechanism.

\section{Magnetotransport}

The Hall resistivity can be expressed as

$$
\rho_{\text {Hall }}=R_{0} B+R_{S} M,
$$

where $R_{0}$ is the ordinary Hall coefficient, $R_{S}$ the anomalous Hall coefficient, and $M$ the magnetization of the film. With $M=\chi H$ and $R_{S}$ proportional to $\rho$, above the Curie temperature $T_{c}$ Eq. (17) becomes

$$
\rho_{\text {Hall }}=R_{H} B,
$$

with

$$
R_{H}=R_{0}+\frac{c \rho \chi}{\mu_{0}},
$$

where $c$ is a constant and $\mu_{0}$ is the permeability of vacuum and $B=\mu_{0} H$. In the paramagnetic region, the susceptibility $\chi$ is given by $C /\left(T-T_{c}\right)$. Figure 12 illustrates that the dominant contribution to the Hall coefficient comes from the extraordinary Hall effect: $\rho / R_{H}$ is proportional to $\left(T-T_{c}\right)$ and $R_{0}$ is zero within experimental error, preventing a determination of the number of charge carriers. Suggested by the resolution of the measurements, the carrier density must exceed $10^{18} \mathrm{~cm}^{-3}$ above $T_{c}$. The above equation can only be used to determine the carrier concentration when the effective-mass approximation is valid. This no longer holds below $T_{c}$, when hopping conduction is involved. ${ }^{24}$

In contrast to the frequently observed positive magnetoresistance for hopping conduction, the resistance of the $\mathrm{Ga}_{1-x} \mathrm{Mn}_{x}$ As layers below $T_{c}$ drops when a magnetic field is applied (Fig. 13). This is an immediate consequence of the 


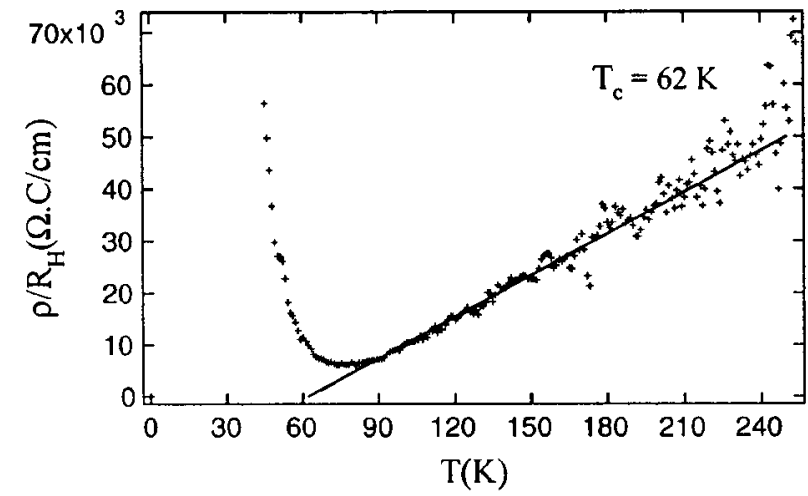

FIG. 12. Graphic illustration of the dominant contribution of the extraordinary Hall effect to the Hall coefficient for $\mathrm{Ga}_{0.940} \mathrm{Mn}_{0.060} \mathrm{As}$ (M271) above $T_{c}\left(T_{c}=62 \mathrm{~K}\right): \rho / R_{H}$ is proportional to the inverse susceptibility at $T>T_{c}$.

antiferromagnetic coupling between the hole and the Mn core. When an external field is applied, all Mn- $h$ complexes align with the field: the $j=3 / 2$ hole magnetic moments are forced antiparallel with the field. Instead of being squeezed (as is the case when no exchange interaction is present) the wave functions expand and the increased overlap results in a negative magnetoresistance. When the magnetic length $\lambda$, defined as

$$
\lambda=\left(\frac{c \hbar}{e H}\right)^{1 / 2}
$$

is small compared to the localization radius $a$ in the absence of a magnetic field, the magnetoresistance in the region of variable-range hopping conduction can be described by ${ }^{19,25}$

$$
\rho(H)=\rho_{0} \exp \left[\frac{C}{\left(\lambda^{2} T\right)^{1 / 3}}\right],
$$

where $C$ is field and temperature independent. This expression is referred to as the strong-field limit because it is only valid when $\lambda \ll a$. The magnetoresistance of different $\mathrm{Ga}_{1-x} \mathrm{Mn}_{x}$ As layers was measured at low temperatures (4, 10 , and $20 \mathrm{~K}$ ) in fields up to $8 \mathrm{~T}$. Fits show that for all measured samples the strong-field limit is fulfilled in the range of 1-8 T (Fig. 13). The negative sign of $C$ confirms the expansion of the wave functions in a magnetic field. The small rise in resistance at low fields corresponds to the alignment of the magnetic domains along the direction of the applied field. As soon as the sample is fully magnetized along the field, the negative magnetoresistance is observed.

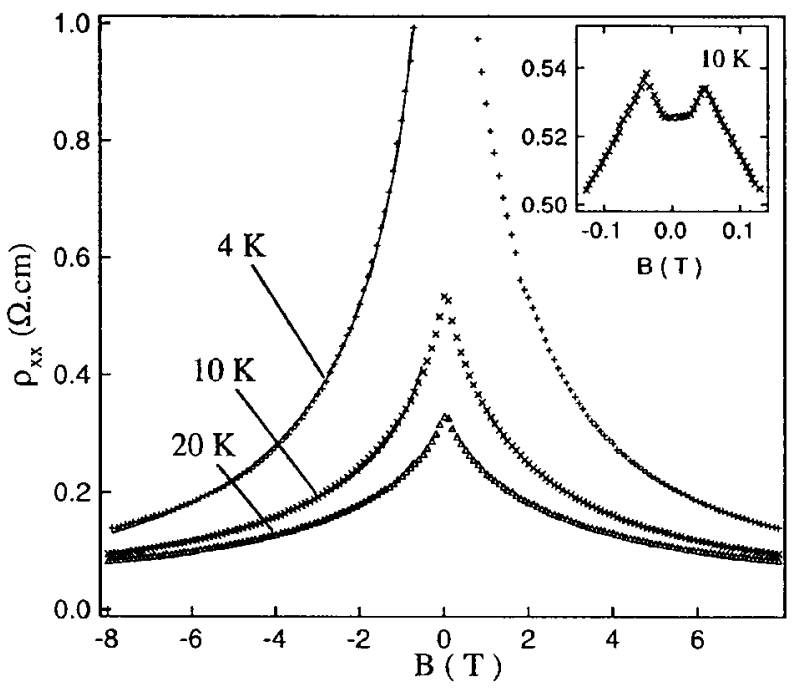

FIG. 13. Magnetoresistance of $\mathrm{Ga}_{0.913} \mathrm{Mn}_{0.087} \mathrm{As}$ (M311) at $T$ $=4,10$, and $20 \mathrm{~K}$. Fits reveal the $\exp \left(H^{1 / 3}\right)$ behavior reminiscent of variable-range hopping in the high-field limit. The inset shows the small rise in resistance due to the aligning of the magnetic domains along the direction of applied field.

\section{CONCLUSION}

$\mathrm{Ga}_{1-x} \mathrm{Mn}_{x} \mathrm{As}$, a new member of the III-V diluted magnetic semiconductor group, has been investigated. The structure of the $\mathrm{Ga}_{1-x} \mathrm{Mn}_{x}$ As lattice as well as the local configuration of $\mathrm{Mn}$ were discussed. The observed paramagnetic to ferromagnetic phase transition at temperatures around $50 \mathrm{~K}$ in all samples $(x=3$ to $9 \%)$ is closely related to the existence of $\mathrm{Mn}-h$ complexes, the latter being formed by the antiferromagnetic coupling between the Mn acceptor core and the hole surrounding it. Resistivity measurements show that above the ordering temperature, transport behavior on both sides of the metal to insulator transition can be observed. Below $T_{c}$, the rising spontaneous magnetization strongly affects the conductivity: a decrease in spin-disorder scattering is accompanied by an increased localization. When the saturation magnetization is reached, variable-range hopping is the main conduction mechanism and an applied magnetic field causes a swelling of the hole wave function, resulting in a negative magnetoresistance.

\section{ACKNOWLEDGMENTS}

This work is supported by the Fund for Scientific Research-Flanders (Belgium) (FWO). L.V.B. and R.B. are supported by the FWO. P.J.W. would like to thank the Deutsche Forschungsgemeinschaft and QUEST (UCSB) for financial support.
${ }^{1}$ J. K. Furdyna, J. Appl. Phys. 64, R29 (1988).

${ }^{2}$ H. Munekata et al., Phys. Rev. Lett. 63, 1849 (1989).

${ }^{3}$ J. De Boeck et al., Appl. Phys. Lett. 68, 2744 (1996).

${ }^{4}$ H. Ohno et al., Appl. Phys. Lett. 69, 363 (1996).

${ }^{5} \mathrm{H}$. Bender et al., in Microscopy of Semiconducting Materials, IOP Conf. Proc. No. 146 (Institute of Physics and Physical Society, London, 1995), p. 293.
${ }^{6}$ Z. Lilienal-Weber, K. M. Yu, and J. Washburn, J. Electron. Mater. 22, 1395 (1993).

${ }^{7}$ C. L. Chan et al., J. Electron. Mater. 22, 1413 (1993).

${ }^{8}$ J. Schneider et al., Phys. Rev. Lett. 59, 240 (1987).

${ }^{9}$ H. Munekata et al., J. Cryst. Growth 127, 528 (1993).

${ }^{10}$ A. Krol et al., Phys. Rev. B 47, 7187 (1993).

${ }^{11}$ J. M. Kikkawa et al., Phys. Rev. B 50, 2003 (1994). 
${ }^{12}$ D. A. Woodbury, and J. S. Blakemore, Phys. Rev. B 8, 3803 (1973).

${ }^{13}$ V. F. Masterov et al., Fiz. Tekh. Poluprovodn. 17, 1259 (1983) [Sov. Phys. Semicond. 17, 796 (1983)].

${ }^{14}$ Th. Frey et al., J. Phys. C 21, 5539 (1988).

${ }^{15}$ X. C. Liu et al., Mater. Sci. Forum 182-183, 627 (1995).

${ }^{16}$ H. Ohno, Mater. Sci. Forum 182-183, 443 (1995).

${ }^{17}$ M. Cohen, Introduction to Magnetic Materials (Addison-Wesley, Reading, MA, 1976).

${ }^{18}$ N. F. Mott, Adv. Phys. 21, 785 (1972).

${ }^{19}$ B. I. Schlovskii and A. L. Efros, Electronic Properties of Doped Semiconductors (Springer-Verlag, Berlin, 1984).
${ }^{20}$ A. Raymond, Application of High Magnetic Fields in Semiconductor Physics, Proceedings of the International Symposium Grenoble, France 1982 (Springer-Verlag, Berlin, 1983), pp. 344-355.

${ }^{21}$ M. A. Dubson and D. F. Holcomb, Phys. Rev. B 32, 1955 (1985).

${ }^{22}$ E. Abrahams et al., Phys. Rev. Lett. 42, 673 (1979).

${ }^{23}$ P. R. Wallace, R. Harry, and M. J. Zuckermann, New Developments in Semiconductors (Noordhoff, The Netherlands, 1973).

${ }^{24}$ C. L. Chien and C. R. Westgate, The Hall Effect and Its Applications (Plenum, New York, 1980).

${ }^{25}$ H. Bottger and V. V. Bryksin, Hopping Conduction in Solids (Akademie-Verlag, Berlin, 1985). 